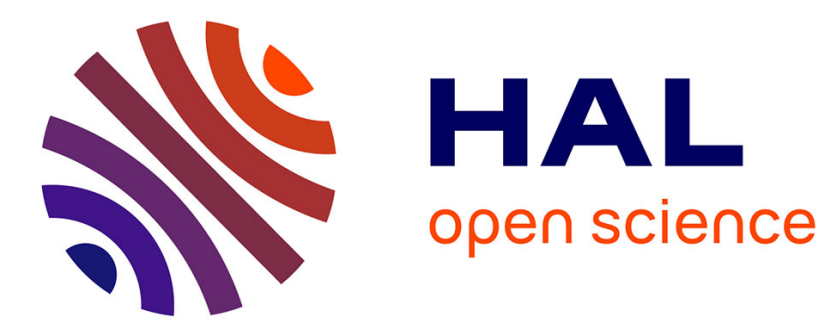

\title{
Thermographic Analysis of Fatigue Dissipation Properties of Steel Sheets
}

B. Berthel, Bertrand Wattrisse, André Chrysochoos, A. Galtier

\section{To cite this version:}

B. Berthel, Bertrand Wattrisse, André Chrysochoos, A. Galtier. Thermographic Analysis of Fatigue Dissipation Properties of Steel Sheets. Strain, 2007, 43 (3), pp.273-279. 10.1111/j.14751305.2007.00349.x . hal-03351263

\section{HAL Id: hal-03351263 https://hal.science/hal-03351263}

Submitted on 22 Sep 2021

HAL is a multi-disciplinary open access archive for the deposit and dissemination of scientific research documents, whether they are published or not. The documents may come from teaching and research institutions in France or abroad, or from public or private research centers.
L'archive ouverte pluridisciplinaire HAL, est destinée au dépôt et à la diffusion de documents scientifiques de niveau recherche, publiés ou non, émanant des établissements d'enseignement et de recherche français ou étrangers, des laboratoires publics ou privés. 


\title{
Thermographic Analysis of Fatigue Dissipation Properties of Steel Sheets
}

\author{
B. Berthel*†, B. Wattrisse*, A. Chrysochoos* and A. Galtier ${ }^{\dagger}$ \\ *Mechanics and Civil Engineering Laboratory, Montpellier II University, CC 48, Place E. Bataillon, 34095 Montpellier Cedex 05, France \\ ${ }^{\dagger}$ Arcelor Research SA, Voie Romaine BP 30320, 57283 Maizières-lès-Metz, France
}

\begin{abstract}
This paper presents several properties of heat source fields accompanying the highcycle fatigue of a dual-phase steel grade. Heat sources were derived from thermal data provided by an infrared focal plane array camera. An especially developed image processing estimates separately the thermoelastic coupling source amplitude and the mean dissipation per cycle. Our experiments underline that dissipation sources are heterogeneous and this forms the beginning of the fatigue test. They also point out a linear evolution of the mean dissipation per cycle as a function of the loading frequency for a given stress range and a given loading ratio.
\end{abstract}

KEY WORDS: dissipation, fatigue, infrared thermography, steel

$\begin{array}{ll}\text { NOTATION } \\ C & \text { Specific heat } \\ d_{1} & \text { Intrinsic dissipation } \\ \bar{d}_{1} & \text { Mean dissipation per cycle } \\ \bar{E}_{\delta \theta} & \text { Mean value of } \delta \theta \\ f_{\mathrm{L}} & \text { Loading frequency } \\ f_{\mathrm{S}} & \text { Sampling frequency } \\ k & \text { Heat conduction coefficient } \\ K & \text { Material conduction tensor } \\ N_{\mathrm{lc}} & \text { Set of loading cycles } \\ N & \text { Set of state variables } \\ p_{i} & i=1, \ldots, 4, \text { second-order polynomials in } x \text { and } y \\ r_{\mathrm{ext}} & \text { External volume heat supply } \\ R_{\sigma} & \text { Loading ratio } \\ s & \text { heat sources } \\ s_{\mathrm{ic}} & \text { Internal coupling sources } \\ s_{\text {the }} & \text { Thermoelastic source } \\ t & \text { Time } \\ T & \text { Absolute temperature } \\ T_{0} & \text { Equilibrium temperature field } \\ W & \text { Specimen width of the virtual test } \\ x & \text { Loading axis }\end{array}$

$\begin{array}{ll}y & \text { Transversal axis } \\ \alpha & \text { Vector gathering the internal variables } \\ \delta \theta & \text { Difference between } \theta^{\exp } \text { and } \theta^{\text {fit }} \\ \Delta \sigma & \text { Stress range } \\ \Delta s_{\text {the }} & \text { Amplitude of the thermoelastic sources } \\ \varepsilon & \text { Linearised strain tensor } \\ \theta & \text { Temperature variations; } \theta=T-T_{0} \\ \theta_{\mathrm{d}} & \text { Dissipative component of the temperature } \\ \theta^{\text {exp }} & \text { signal } \\ \theta^{\text {fit }} & \text { Experimentally measured temperature } \\ \theta_{\text {the }} & \text { Local fitting function of the temperature } \\ & \text { Thermoelastic component of the temperature } \\ \rho & \text { signal } \\ \sigma_{\delta \theta} & \text { Mass density } \\ \sigma_{\mathrm{y}} & \text { Standard deviation of } \delta \theta \\ \tau & \text { Yield stress } \\ \tau_{\mathrm{th}}^{2 \mathrm{D}} & \text { Period of the virtual test } \\ & \text { Time constant that characterises the perpen- } \\ & \text { dicular heat exchanges between front and back }\end{array}$

\section{Introduction}

The classical fatigue characterisation of materials and structural components requires the statistical processing of numerous mechanical cyclic tests which are time consuming and particularly expensive. Alternative experimental approaches have been developed for several years to provide reliable fatigue characteristics more rapidly. Among

these, the thermal methods, based on the analysis of the self-heating during a stepwise loading fatigue test, must be mentioned [1-5]. These methods claim that the change of the heating regime, observed at a certain stress range, is related to the fatigue limit of the material. Although realistic estimates of this limit were sometimes obtained, the thermal approaches often led to questionable results [6]. Indeed, the empirical 
construction of the threshold stress evaluated by the thermal methods and its physical meaning are not sufficiently satisfactory. This stress cannot be a proper fatigue limit as it corresponds to the appearance of persistent slip bands and not to the macrocrack creation [7].

In previous studies we also underlined that the direct use of the specimen temperature as fatigue indicator is not always reliable because the temperature variation is not intrinsic to the material behavior $[8,9]$. It depends actually not only on the diffusion properties (material effect) but also on thermal boundary conditions and on the distribution of heat sources (structure effects).

In this analysis, the fatigue phenomena were therefore studied using a calorimetric approach. The aim was to assess the heat source fields created during the fatigue test. The heat sources are derived from thermal images provided by an infrared camera using a local expression of the heat equation. A specific thermal image processing was developed to separately estimate the dissipated energy associated with the irreversible evolutions of the microstructural defects and the thermoelastic coupling sources induced by the reversible thermal expansion of the crystalline network.

The paper is composed as follows: the thermal diffusion model used to compute the heat source is first introduced. The experimental set-up and the image-processing method are then presented together with some numerical simulations in order to illustrate not only the consistency but also the limits of the heat source computation in the case of noisy, undersampled thermal signals. Several experimental results obtained on a dual-phase steel are finally analysed.

\section{Calorimetric Balance and Definition of Heat Sources}

The fatigue is considered as a dissipative, quasi-static thermomechanical process. The equilibrium state of each volume of material is then described by means of a set of $N$ state variables. The chosen set of state variables is made of: the absolute temperature $T$, the linearised strain tensor $\varepsilon$ and $N-2$ scalar components $\alpha_{1}, \alpha_{2}, \ldots \alpha_{N-2}$ of the vector $\alpha$ gathering the internal variables. These describe the macroscopic effects of complex, coupled microstructural phenomena. Within the framework of Generalised Standard Materials [10], the local heat diffusion equation can be written as:

$\rho C \dot{T}-\operatorname{div}(K \operatorname{grad} T)=d_{1}+s_{\text {the }}+s_{\text {ic }}+r_{\text {ext }}$ where $\rho$ is the mass density, $C$ the specific heat, $K$ the material conduction tensor. The left-hand side of Equation (1) is a differential operator applied to $T$, while the right-hand side groups all the possible heat sources linked with the deformation process. They are in turn the intrinsic dissipation $d_{1}$, the thermomechanical coupling sources made of the thermoelastic source $s_{\text {the }}$ and the other internal coupling sources $s_{\mathrm{ic}}$, and the external volume heat supply $r_{\text {ext }}$.

To perform the heat source evaluation by using infrared images given by an infrared camera the following hypotheses were formulated [11]:

1 The heat conduction is isotropic and the related coefficient $k$ remains constant during the test.

2 The density $\rho$ and the specific heat $C$ are material constants, independent from the internal state.

3 The convective terms, included in the material time derivation, are neglected.

4 The external heat supply $r_{\text {ext }}$ (here only due to radiation heat exchanges) is time-independent.

Consequently, the equilibrium temperature field $T_{0}$ verifies:

$-k \Delta T_{0}=r_{\mathrm{ext}}$.

Moreover, it is assumed that the temperature variations $\theta=T-T_{0}$ induced by fatigue mechanisms remain too small to have any influence on the microstructural state. In other words, we assume that $s_{\mathrm{ic}}=0$.

Under these hypotheses the local heat equation (1) can be rewritten in the following simplified form:

$\rho C \frac{\partial \theta}{\partial t}-k \Delta \theta=d_{1}+s_{\text {the }}=s$

where $s$ henceforth denotes the overall volume heat source.

By integrating Equation (3) over the sample thickness $(\mathrm{Oz}$ direction in Figure 1$)$ and assuming that the depth-wise averaged temperature field $\theta(x, y, t)$ is very close to the temperature distribution measured at the specimen surface (small sample thickness and high material diffusivity), the following equation can be obtained:

$\frac{\partial \theta}{\partial t}+\frac{\theta}{\tau_{\text {th }}^{2 \mathrm{D}}}-\frac{k}{\rho C}\left(\frac{\partial^{2} \theta}{\partial x^{2}}+\frac{\partial^{2} \theta}{\partial y^{2}}\right)=\frac{s}{\rho C}$

where $s=s(x, y, t)$ is now the 2D heat-source distribution - for the sake of simplicity the same notation is kept - and $\tau_{\text {th }}^{2 \mathrm{D}}$ is a time constant that characterises the perpendicular heat exchanges (by convection and radiation) between front and back specimen faces and the surroundings. Let us note that the sources divided 


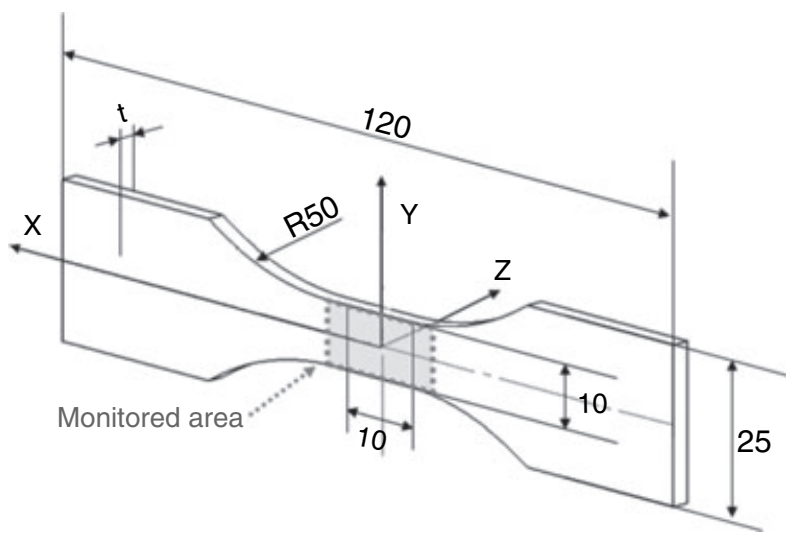

Figure I: Specimen drawing (dimension in $\mathrm{mm}$ and $t$ the sheet thickness)

by $\rho C$ are then expressed in ${ }^{\circ} \mathrm{C} \mathrm{s}^{-1}$. This operation allows to define, for each type of source, an equivalent heating speed associated with a monotonous thermal process in adiabatic conditions and facilitates comparison between the coupling and dissipative sources.

\section{Experimental Set-up}

The material under examination is the DP600 steel (hot rolled) produced by Arcelor Research (Maizières-lèsMetz, France) (flat dual-phase carbon steel). The fatigue tests were conducted using a servo-hydraulic machine equipped with a c. $25 \mathrm{kN}$ load cell. Thin flat specimens were used with a gauge part of $10 \mathrm{~mm}$ length, $10 \mathrm{~mm}$ width and $2.6 \mathrm{~mm}$ thickness (Figure 1).

The experimental setup included a focal plane array infrared camera (IRFPA Cedip MW manufactured by Cedip Infrared systems, Croissy-Beaubourg, France). The maximum frame-rate of the IR camera is 250 images per second, with an image resolution of $64 \times 120$ pixels.

During the tests, the infrared device is placed in front of the specimen, with the lens axis being fixed and perpendicular to the specimen surface. The spatial resolution is approximately $0.13 \mathrm{~mm}$ per pixel. The fatigue tests are carried out by submitting series of loading blocks. Each block consisted of 20000 cycles performed at constant loading frequency $f_{\mathrm{L}}$ ( $3 \leq f_{\mathrm{L}} \leq 50 \mathrm{~Hz}$ ), constant load ratio $R_{\sigma}$, and constant stress range $\Delta \sigma$. At the end of each loading block, the thermal equilibrium has been awaited before the next block starts.

\section{Computation of Heat Sources}

The purpose of the thermal data processing is to estimate the mean amplitude of the thermoelastic sources $\Delta s_{\text {the }}$ and the mean dissipation $\bar{d}_{1}$ per cycle over a set of $N_{\text {lc }}$ loading cycles. The construction of the heat source distribution via Equation (4) requires the evaluation of partial derivative operators applied to noisy digital signals. To improve the signalto-noise ratio, it is then necessary to reduce the noise amplitude without modifying the spatial and temporal thermal gradients. Among several possible methods, a special local least-squares fitting of the thermal signal is considered in this work. The temperature approximation function accounts for the spectral properties of the underlying heat sources. Moreover, the linearity of Equation (4) and that of the respective boundary conditions enabled us to separately analyse the influence of thermoelastic and dissipative heat sources with $\theta_{\text {the }}$ and $\theta_{\mathrm{d}}$ respectively denoting the thermoelastic and dissipative components of the temperature signal.

The local fitting function $\theta_{\mathrm{f}}$ of the temperature charts is chosen as in [12]:

$$
\begin{aligned}
\theta^{\mathrm{fit}}(x, y, t)= & p_{1}(x, y) t+p_{2}(x, y)+p_{3}(x, y) \cos \left(2 \pi f_{\mathrm{L}} t\right) \\
& +p_{4}(x, y) \sin \left(2 \pi f_{\mathrm{L}} t\right)
\end{aligned}
$$

where the trigonometric time functions describe the periodic part of the thermoelastic effects while the linear time function takes transient effects due to heat losses, dissipative heating and possible drifts in the equilibrium temperature into account.

The functions $p_{i}(x, y), i=1, \ldots, 4$, are second-order polynomials in $x$ and $y$. These polynomials enable us to account for the possible spatial heterogeneity of the source pattern. The efficiency of the local fitting of the temperature field can be estimated by the temperature difference $\delta \theta_{i j k}$ between the noisy measurements $\theta_{i j k}^{\exp }=\theta^{\exp }\left(x_{i}, y_{j}, t_{k}\right)$ and the fitted values $\theta_{i j k}^{\mathrm{fit}}=\theta^{\mathrm{fit}}\left(x_{i}, y_{j}, t_{k}\right)$. Let us take for example the following standard situation where the approximation data regroups $(21 \times 21)$ pixels $\times 57$ frames. For such an example, the mean error $\bar{E}_{\delta \theta}=\langle\delta \theta\rangle$ is about 0 (centred Gaussian distribution), while the standard deviation $\sigma_{\delta \theta}$ is about $0.030{ }^{\circ} \mathrm{C}$. These estimates were performed by using a set of experimental noisy thermal data extracted from a real fatigue test. Besides, the noise in the infrared measurements is characterised by a centred Gaussian distribution of standard deviation $0.026^{\circ} \mathrm{C}$. Both results show that the approximation function set is convenient to correctly construct a denoised temperature signal. Nevertheless, the efficiency of the fitting must also be checked in terms of heat source computations, these latter being mainly related to the temperature derivatives.

To test the capabilities of the image processing, a direct $3 \mathrm{D}$ thermal problem is first solved for a given 
3D distribution of dissipative and thermoelastic sources. Naturally, realistic initial and boundary conditions have also to be fixed. Because of small temperature variations, linear Fourier conditions are systematically chosen to model heat exchanges between the sample and the surrounding air and between the sample and the grips of the testing machine. The temperature field, as determined by the solution of the (direct) partial derivative problem, is finally used to test the image-processing method (inverse problem), with the reconstructed distribution of heat sources being compared with the given initial one.

In Figures 2 and 3, comparisons between given and computed heat sources are proposed. The material, geometrical and optical parameters were chosen close to their experimental values. Besides, the evolution of the surface temperature field is derived from the following parameters. The heat sources are randomly distributed within the sample volume and are time-independent. The thermoelastic source amplitude and the dissipation intensity varied within $(100 \pm 10)^{\circ} \mathrm{C} \mathrm{s}^{-1}$ and $(0.1 \pm 0.1)^{\circ} \mathrm{C} \mathrm{s}^{-1}$, respectively. This should reproduce the heterogeneous character of the fatigue process and its slow evolution during the test for fixed loading conditions. We have also taken into account the orders of magnitude of the dissipative and coupling sources $\left(\bar{d}_{1} / \Delta s_{\text {the }} \approx 10^{-3}\right)$ experimentally evidenced. We finally superimposed on the temperature variations a noise in accordance with the metrological analysis of the camera signal (Gaussian white noise, std $=0.026^{\circ} \mathrm{C},[13]$ ). The loading frequency $f_{\mathrm{L}}$ and sampling frequency $f_{\mathrm{S}}$ were 50 and $19 \mathrm{~Hz}$ respectively. Both values correspond to standard loading and sampling conditions used during the camera shots.

To illustrate the capabilities of the heat source computation method, the temperature of four pixels

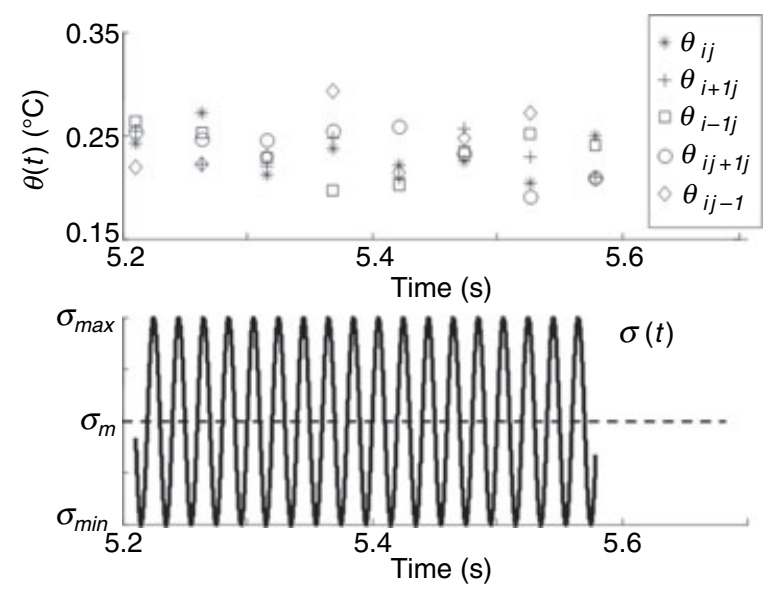

Figure 2: Examples of sampled thermal data
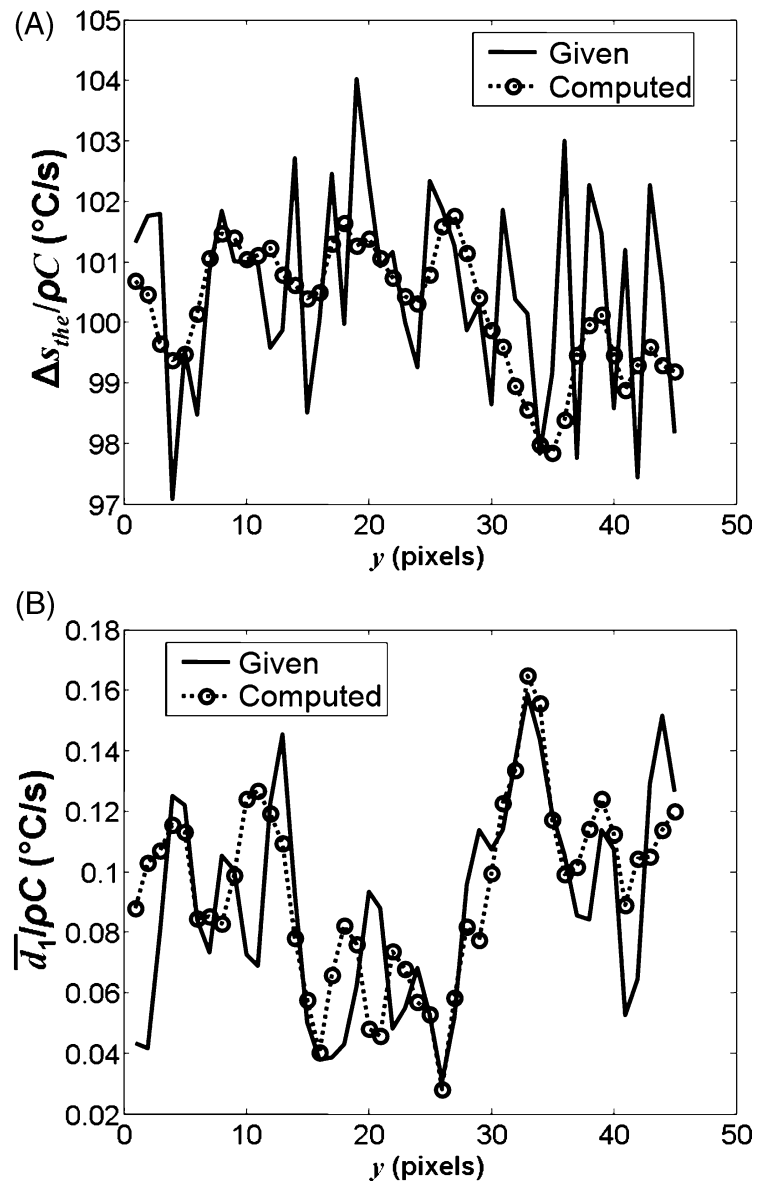

Figure 3: Comparison between given and computed values: (A) thermoelastic source range; (B) dissipative source

surrounding a fifth one is plotted as a function of time in Figure 2. The analogical loading signal is also drawn to underline the under sampling level allowed by the image processing. The reliability of the source computation is checked by comparing the given heat source distribution with the one resulting from the data processing in the case of a noisy, under-sampled thermal signal.

In Figure $3 \mathrm{~A}$ and $\mathrm{B}$ the longitudinal profiles of $\Delta s_{\text {the }}(x=W / 2, y, \tau / 2)$ and $\bar{d}_{1}(x=W / 2, y, \tau / 2)$, respectively, both captured in the middle of the sample width $W / 2$ at the half-period $\tau / 2$ of the virtual test are plotted. Despite the different orders of magnitude of both source types, data processing is able to quantitatively detect different heat source intensity and to qualitatively reproduce the global trends in their distribution. The method may naturally become unstable and fail for smaller dissipation intensity or noisier thermal data.

To illustrate these limits, we chose an example of data processing with the same previous conditions, but with dissipation intensities 5 times smaller. Figure 4A shows that the data processing still computes correct thermoelastic source amplitudes. 


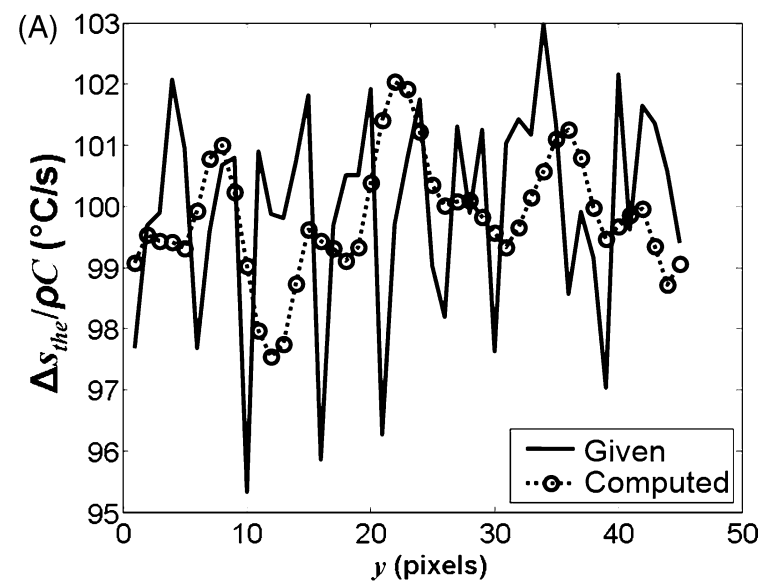

(B)

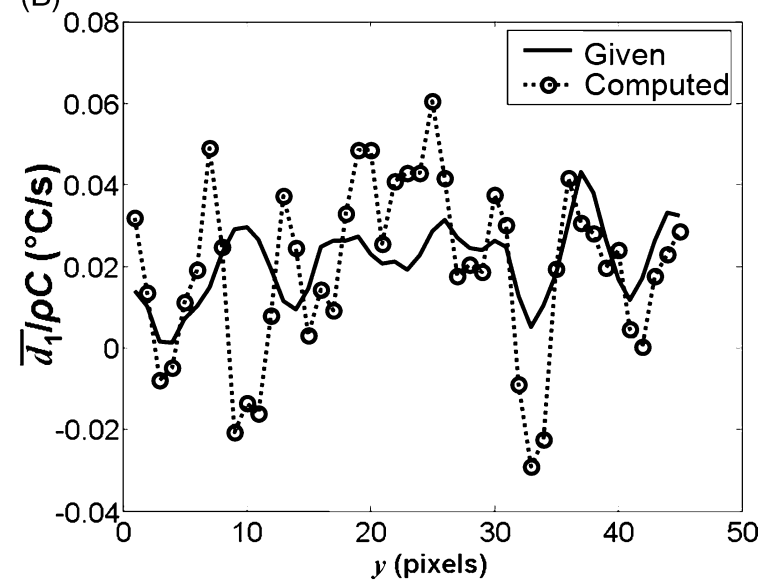

Figure 4: Comparison between given and computed values: (A) thermoelastic source range; (B) dissipative source

Nevertheless, the method gives corrupted dissipation values, as indicated in Figure $4 \mathrm{~B}$ where some values are even negative, which is physically unacceptable.

\section{Examples of Experimental Results}

The image processing then allows us to analyse noisy thermal images provided by the infrared camera for a wide range of experimental conditions. In what follows, properties of thermoelastic source amplitude and mean dissipation during high-cycle fatigue test performed on a dual-phase 600 steel grade are successively presented. The selected monitoring area is shown in Figure 1.

\section{Properties of $\Delta s_{\text {the }}$}

Until the crack onset, we observed quasi-uniform patterns of thermoelastic source amplitudes as expected for a simple tension-compression test [14]. Besides, the experiments showed that $\Delta s_{\text {the }}$ increased linearly with $\Delta \sigma$ and $f_{\mathrm{L}}$ as predicted by linear thermoelasticity.
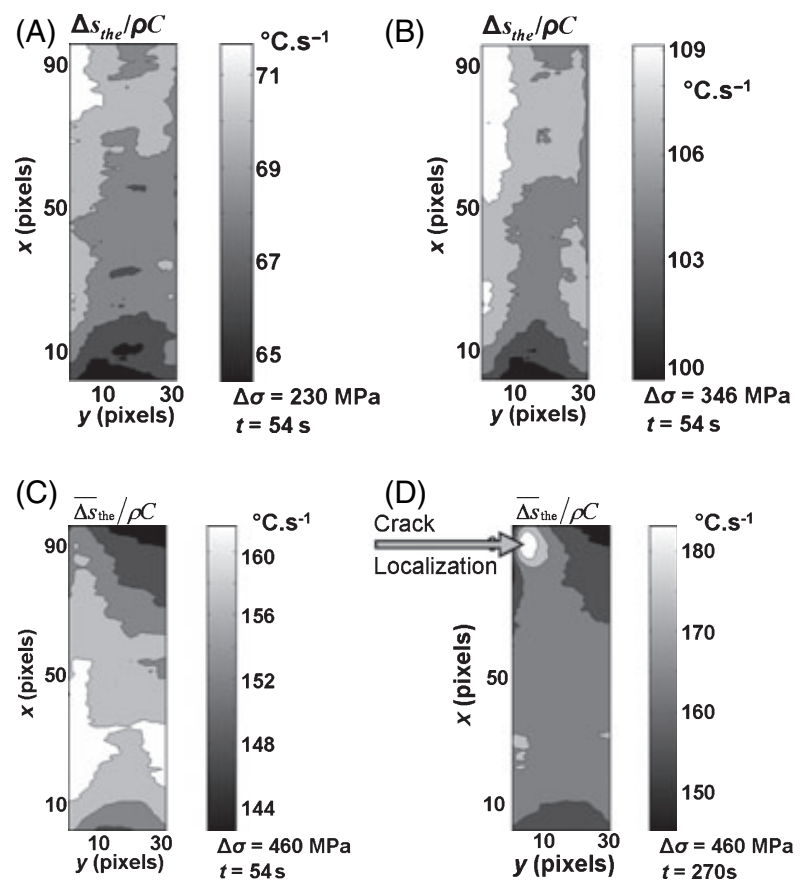

Figure 5: Patterns of $\Delta s_{\text {the }}(x, y)$ for $4 \Delta \sigma$ (i.e. loading block), $f_{L}=50 \mathrm{~Hz}$ and $R_{\sigma}=0$

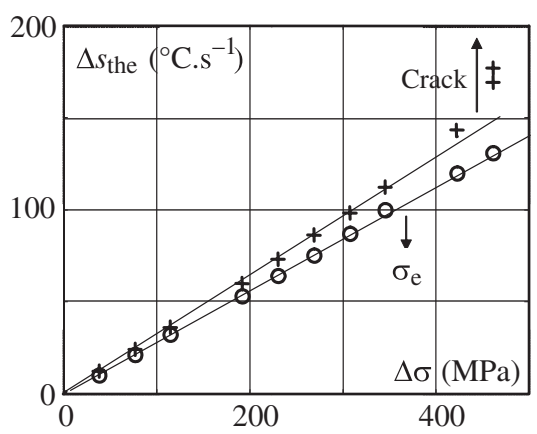

Figure 6: (o) $\min /(+) \max$ values of $\Delta s_{\text {the }}$ versus $\Delta \sigma$

In the test shown below, the specimen was slightly misaligned in order to induce slight gradients in the distribution of thermoelastic sources. As previously mentioned, the test was made of loading blocks at different $\Delta \sigma$ with $R_{\sigma}=0$ and $f_{\mathrm{L}}=50 \mathrm{~Hz}$.

Despite the out-of-axis loading, Figure 6, derived from Figure 5, shows that the $\mathrm{min} / \mathrm{max}$ values of $\Delta s_{\text {the }}(x, y)$ increase linearly with $\Delta \sigma$ until the yield stress is reached $\left(\sigma_{\mathrm{y}}=360 \mathrm{MPa}\right)$. This means that the 'elastic' stress field changes with $\Delta \sigma$ in a homothetic way so that the overall stress range remains representative of the stress pattern, as it can be qualitatively observed between Figure 5A and Figure 5B. We arbitrarily chose to plot the $\Delta s_{\text {the }}(x, y)$ pattern at time $t=54 \mathrm{~s}$, the distribution being approximately constant throughout the loading block.

During the next blocks (Figure 5C,D), plasticity develops, inducing a stress redistribution. The presence 


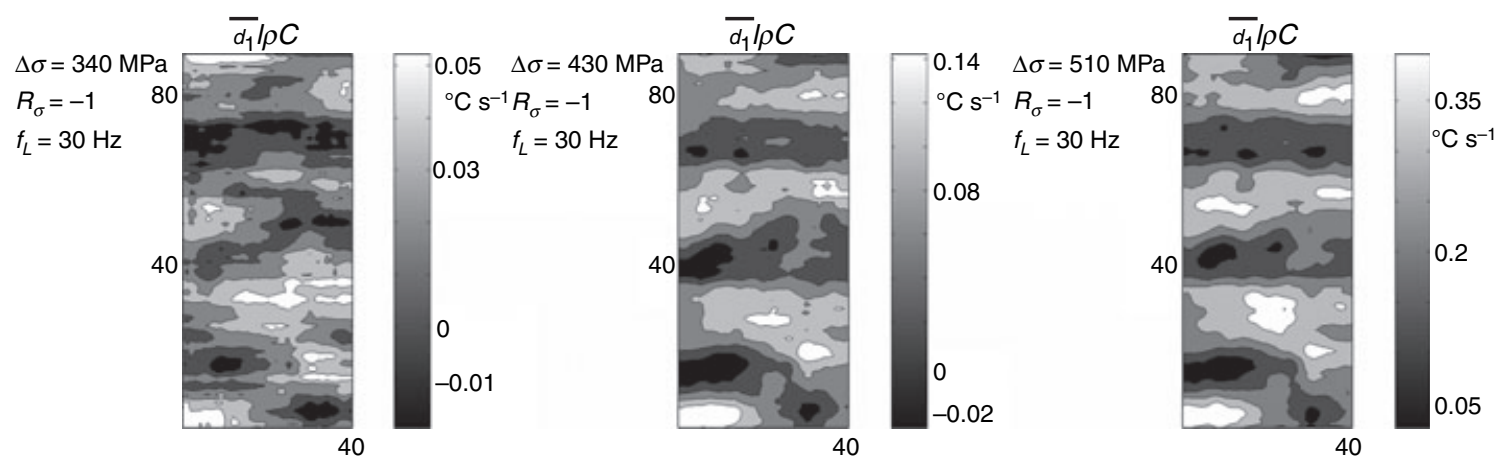

Figure 7: Dissipation intensity versus stress range

of stress concentration may explain why the maximal value of $\Delta s_{\text {the }}$ is no longer proportional to $\Delta \sigma$. The loss of linearity is naturally less important in regions where the stress gradients are softer. Before the crack onset, Figure 5C (plotted for $t \approx 54 \mathrm{~s}$ ) shows a homothetic pattern of $\Delta s_{\text {the }}(x, y)$ compared with Figure 5A,B. In Figure 5D, corresponding to the crack onset $(t \approx 270 \mathrm{~s})$, a localisation of thermoelastic sources can be observed at the crack tip. The effect of the crack propagation at constant stress range on the thermoelastic source can be observed on the right side of Figure 6.

\section{Properties of dissipation}

Figure 7 shows the pattern of mean dissipation per cycle calculated at different stress ranges (i.e. for different loading blocks). During a block, this heterogeneous pattern remains constant as long as a fatigue crack does not initiate. Contour plots in Figure 7 also show that areas where the dissipation is high (respectively low) remain approximately the same from one block to another even if the dissipation naturally increases with the stress range. During a (last) loading block, we observed a localised zone of high dissipation several hundreds of cycles before the crack initiation. This zone indeed corresponds to the location of the crack inception. Moreover, we also noticed that this zone always corresponds to a candidate zone where the dissipation level is high since the beginning of the fatigue test (i.e. for low stress ranges.)

Besides, Figure 8 shows examples of dissipation evolution associated with zones of the same specimen as a function of $\Delta \sigma$ and $f_{\mathrm{L}}$. During this test performed with a load ratio of -1 , the stress range was increased from 170 to $500 \mathrm{MPa}$ and for each load level several frequencies $f_{L}$ were used from 9 up to $30 \mathrm{~Hz}$. Values of dissipation in the first location (Figure 8A) are twice smaller than the one measured in the second area (Figure $8 \mathrm{~B}$ ). First, it can be noticed that the shape of both surfaces $\bar{d}_{1}\left(\Delta \sigma, f_{L}\right)$ is very

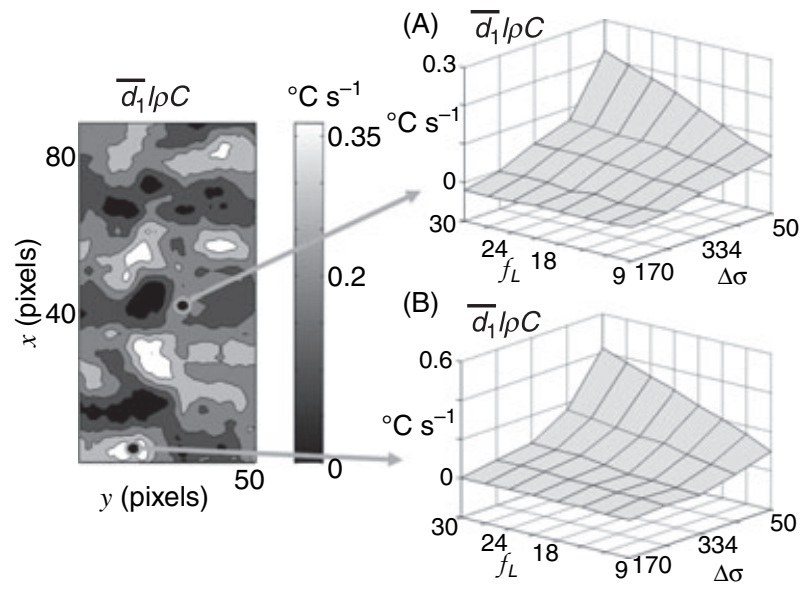

Figure 8: Dissipation evolution within two different material zones

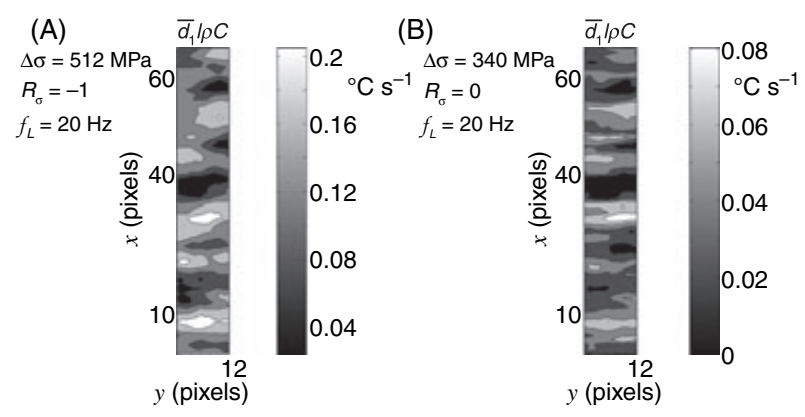

Figure 9: Dissipation pattern: (A) $R_{\sigma}=-1, \Delta \sigma=512 \mathrm{MPa}$; (B) $R_{\sigma}=0, \Delta \sigma=340 \mathrm{MPa}$

similar. At constant stress, we also underline that $\bar{d}_{1}$ increases with the load frequency $f_{\mathrm{L}}$ in a quasi-linearly way for both zones and for all stress ranges.

Finally, the same specimen was used to perform two tests with different loading ratio $\left(R_{\sigma}=-1\right.$ and $\left.R_{\sigma}=0\right)$ and for a given loading frequency $\left(f_{L}=\right.$ $20 \mathrm{~Hz}$ ). The corresponding dissipation patterns, shown in the Figure 9, are roughly the same. Moreover, if the specimen is rotated up side down, the dissipation field follows this rotation. Consequently, this test shows that the distribution of dissipation is actually related to material microstructure and not, for example, to loading misalignment. 


\section{Concluding Comments}

In this paper, an infrared image-processing method that enables to estimate the coupling and dissipative sources accompanying the fatigue is presented. Therefore a local expression of the heat equation is used. The frequency spectra of the thermoelastic amplitude and of the mean dissipation per cycle allow us to compute both sources with the same thermosignal. As expected, the sensitivity of the method is strongly dependent on the intensity and distribution of the investigated heat sources but also on the signal-to-noise ratio of the temperature measurements. Numerical tests are shown to illustrate the capabilities and limits of the calorimetric approach. In particular the method fails for very low dissipation levels $\left(<0.03{ }^{\circ} \mathrm{C} \mathrm{s}^{-1}\right)$ and/or for low signal-to-noise ratio.

Applications to Dual Phase 600 steel sheet show a linear evolution of the thermoelastic source amplitude as a function of the stress range and the loading frequency in agreement with the linear thermolasticity theory. We also observed a heterogeneous distribution of the dissipation since the beginning of the test. This distribution remains time independent and does not change with the stress range and/or the loading ratio until a fatigue crack initiate. Besides, the linear evolution of the dissipation intensity with the loading frequency is underlined.

In terms of structure design, this method may be useful to detect fatigue zones in homogeneous loading areas. In terms of material analysis, the dissipation distribution can be combined with kinematical measurement fields to build up a complete energy balance [15]. We are now developing a method combining infrared thermography and digital image correlation using a synchronisation system. The dissipative source will can be compared with the hysteresis area of the stress-strain curve. Our ultimate objective is to determine both stored and dissipated energy patterns useful to identify fatigue kinetics of materials.

\section{ACKNOWLEDGEMENTS}

The authors would like to thank Arcelor Research SA and Nippon Steel Corporation for their technical and financial support during this study.

\section{REFERENCES}

1. Galtier, A. (1993) Contribution à l'étude de l'endommagement des aciers sous sollicitations uni ou multiaxiale. Thesis, ENSAM, Bordeaux, France.

2. Luong, M. P. (1998) Fatigue limit evaluation of metals using an infrared thermographic technique. Mech. Mater. 28, 155-163.

3. La Rosa, G. and Risitano, A. (2002) Thermographic methodology for rapid determination of the fatigue limit of materials and mechanical components. Int. J. Fatigue 22, 65-73.

4. Liaw, Y. B., Wang, P. K., Jiang, L, Huang, J. Y., Kuo, R. C. and Huang, J. C. (2001) Thermographic investigation of the fatigue behaviour of reactor pressure vessel steels. Mater. Sci. Eng. A 314, 131-139.

5. Krapez, J. C., Pacou, D. and Gardette, G. (2000) Lock-in thermography and fatigue limit of metals. Proc. QIRT'2000, Reims, France, 277-282.

6. Krapez, J. C. and Pacou, D. (2002) Thermography detection of damage initiation during fatigue tests. Proc. SPIE Thermosense XXIV, Orlando, FL, USA, 4710, 435-449.

7. Cugy, P. and Galtier A. (2002) Microplsaticity and temperature increase in low carbon steels. Proc. 8th Int. Fatigue Conf., Stockholm, Sweden, 549-556.

8. Mabru, C. and Chrysochoos, A.(2001) Dissipation et couplages accompagnant la fatigue des matériaux, métalliques. Proc Photomécanique' 01, Poitiers, France, 375-382.

9. Boulanger, T., Chrysochoos, A., Mabru, C. and Galtier, A. (2004) Calorimetric and thermoelastic effects associated with the fatigue behavior of steels. Int. J. Fatigue 26, 221229.

10. Germain, P., Nguyen, Q. S. and Suquet, P. (1983) Continuum thermodynamics. J. Appl. Mech. 50, 1010-1020.

11. Chrysochoos, A. and Louche, H. (2000) An infrared image processing to analyse the calorific effects accompanying strain localisation. Int. J. Eng. Sci. 38, 1759-1788.

12. Chrysochoos, A., Boulanger, T., Wattrisse, B. and Morabito A. E. (2004) Analyse calorimétrique de la fatigue des métaux. Proc. Photomécanique'04, Albi, France, 401-408.

13. Honorat, V., Moreau, S., Muracciole, J.-M., Wattrisse, B. and Chrysochoos, A. (2005) Calorimetric analysis of polymer behaviour using a pixel calibration of an IRFPA camera. Int. J. Quant. Infrared Thermography 2, 153-172.

14. Berthel, B., Chrysochoos, A., Wattrisse, B. and Galtier, A. (2006) Analyse thermographique du comportement en fatigue de l'acier DP600. Proc. Colloque National MECAMAT, Aussois, France, 306-310.

15. Chrysochoos, A., Muracciole, J.-M. and Wattrisse, B. (2000) Experimental analysis of strain and damage localization. Proc. Symp. Continuous Damage and Fracture, Cachan, France, 41-51. 